\title{
Exosomes from mesenchymal stem cells expressing microribonucleic acid-125b inhibit the progression of diabetic nephropathy via the tumour necrosis factor receptor-associated factor 6/Akt axis
}

\author{
Xia Cai, Fang Zou, Rui Xuan and Xiao-Yang Lai \\ Department of Endocrinology, The Second Affiliated Hospital of Nanchang University, Nanchang 330006, Jiangxi Province, \\ P.R.China
}

\begin{abstract}
Diabetic nephropathy (DN) seriously threatens the health of patients with diabetes. Moreover, it has been reported that mesenchymal stem cell (MSC)-derived exosomal miRNAs can modulate the progression of multiple diseases, including DN. It has been suggested that miR-125b is involved in DN. However, the biological functions of exosomal miRNAs, especially miR-125b, in DN are still unclear. To establish a DN model in vitro, we used a model of human embryonic kidney epithelial cells (HKCs) injury induced by high glucose (HG). Then, miR-125b was delivered to the model cells in vitro via MSC-derived exosomes (MSC-Exos), and the effect of exosomal miR-125b on HKCs apoptosis was evaluated by flow cytometry. qRT-PCR or western blotting was performed to measure miR-125b or tumour necrosis factor receptor-associated factor 6 (TRAF6) expression in HKC. The effect of MSC-Exos on HKCs apoptosis after miR-125b knockdown was determined by flow cytometry. Moreover, dual-luciferase reporter assays were used to determine the targeting relationship between miR-125b and TRAF6 in HKCs. Our data revealed that MSC-Exos increased HG-induced autophagy in HKCs and reversed HKCs apoptosis. Moreover, our study found that miR-125b was enriched in MSC-Exos and directly targeted TRAF6 in HKCs. In addition, exosomally transferred miR-125b inhibited the apoptosis of HG-treated HKCs by mediating Akt signalling. In summary, MSC-derived exosomal miR-125b induced autophagy and inhibited apoptosis in HG-treated HKCs via the downregulation of TRAF6. Therefore, our study provided a new idea for DN treatment.
\end{abstract}

Key words: miR-125b, Autophagy, Apoptosis, Diabetic nephropathy

DIABETIC NEPHROPATHY (DN) is a common diabetic complication that results from permanent uncontrolled diabetes, and it usually causes end-stage renal diseases $[1,2]$. Moreover, previous studies have indicated that the induction of autophagy and inhibition of apoptosis could lead to the suppression of DN progression $[3,4]$. Since DN seriously threatens the health of patients with diabetes, it is necessary to find new strategies for inducing autophagy and inhibiting apoptosis in DN.

Exosomes are membranous vesicles that regulate recipient cell functions by transferring mRNAs, miRNAs and soluble proteins in functional forms $[5,6]$. Moreover, mesenchymal stem cell-derived exosomes (MSC-

Submitted Oct. 10, 2020; Accepted Feb. 15, 2021 as EJ20-0619 Released online in J-STAGE as advance publication May 21, 2021 Correspondence to: Xiao-Yang Lai, Department of Endocrinology, The Second Affiliated Hospital of Nanchang University, No. 1, Minde Road, Nanchang 330006, Jiangxi Province, P.R.China.

E-mail: laixyang60@sina.com
Exos) have been reported to exhibit suppressive effects on cancer and inflammation [7-9]. Wang D et al. showed that MSC-Exos targeting miR-125b could inhibit neointimal hyperplasia by activating myosin IE [10]. Moreover, the function of exosomes in cells provides multiple therapeutic applications, including cell growth and tissue injury repair following myocardial ischaemia reperfusion [11], neurological stroke [12] and inflammation [13]. In recent years, research on the mechanism of miRNA transfer, including exosomes, has attracted increasing attention $[14,15]$. Zhu M et al. found that high glucose (HG)-induced exosomes derived from macrophages promoted macrophages to alleviate injury in kidney tissues during DN [16]. Furthermore, many studies have confirmed the function of exosomal miRNAs in DN [17, 18]. For example, urinary exosomal miR-21-5p and miR-30b-5p participate in DN [18]. MSCs can deliver exogenous miR-let7c via exosomes to attenuate renal fibrosis [19]. Furthermore, MSCs are known to have therapeutic effects on DN [20]. These data suggested that 
MSC-derived exosomal miRNAs might act as key mediators in DN. However, the function of exosome-mediated miRNA transfer in DN is largely unknown.

Previous studies have revealed some miRNAs that play important roles in the progression of DN. For instance, miR-143-3p can mediate the development of DN by targeting TGF- $\beta 1$ [21]. In addition, Wang $\mathrm{F}$ et al. revealed that miR-21 can attenuate the symptoms of DN [22]. Moreover, miR-125b has been shown to be downregulated in diabetic neuropathy (DNP) [23]. Reports have indicated that miR-125b is downregulated in the exosomes found in the urine of patients with diabetes and diabetic kidney disease [18]. In addition, exosomal miR-125b derived from cardiovascular endothelial cells can induce cell autophagy to inhibit the progression of myocardial infarction [24]. However, the effect of MSCExo-transferred miR-125b on DN remains unclear.

Tumour necrosis factor receptor-associated factor 6 (TRAF6) is considered a downstream modulator of IL-1 $\beta$, which is involved in inflammation [25]. TRAF6 has been reported to act as a key mediator in DN [26, 27]. Moreover, in a study of DN-induced podocyte damage, it was found that inhibition of the Akt signalling pathway could lead to autophagy and inhibit cell apoptosis $[28,29]$. In addition, it has been reported that TRAF6 regulates Akt signalling in prostate hyperplasia and promotes disease progression [30-32]. Based on this background, we conclude that the TRAF6 and Akt signalling pathways may play a crucial role in $\mathrm{DN}$, and we speculate that TRAF6 affects the development of DN by regulating the Akt axis in DN.

In this study, we sought to confirm the biological function of MSC-Exos containing miR-125b in the progression of DN. We constructed an in vitro model of DN and added MSC-Exos containing miR-125b to detect changes in autophagy and apoptosis of the model cells. Our study confirmed that exosomes from MSCs expressing miR-125b significantly induced the autophagy and inhibited the apoptosis of HG-treated HKCs via mediation of the TRAF6/Akt axis. These findings will provide a new idea for DN treatment.

\section{Material and Methods}

\section{Cell culture and reagents}

Bone marrow MSCs were extracted as previously described [33]. Total bone marrow cells were harvested from rats. The cells were then cultured in DMEM/F12 (HyClone, Logan, UT, USA) supplemented with 10\% foetal bovine serum (FBS, Gibco, Carlsbad, CA, USA) at $37^{\circ} \mathrm{C}$ in $5 \% \mathrm{CO}_{2}$.

Human embryonic kidney epithelial cells (HKCs) were purchased from ATCC (Manassas, VA, USA) and maintained in RPMI-1640 medium (Thermo Fisher Scientific, Waltham, MA, USA) containing 10\% FBS, $1 \%$ penicillin (Thermo Fisher Scientific) and 1\% streptomycin (Thermo Fisher Scientific) at $37^{\circ} \mathrm{C}$ and $5 \% \mathrm{CO}_{2}$. To establish an in vitro model of $\mathrm{DN}$, HKCs were treated with high D-glucose (HG, Pepro Tech, Rocky Hill, NJ, USA) at a concentration of $35 \mathrm{mM}$ for $48 \mathrm{~h}$. Furthermore, mannitol, low glucose (LG) and lysosome inhibitor (Baf A1) were obtained from Pepro Tech (Rocky Hill, NJ, USA).

\section{Tissue collection}

Fifteen pairs of DN tissues and adjacent normal tissues were collected from the Second Affiliated Hospital of Nanchang University between August 2019 and December 2019. The clinical and pathological data and tissues of these patients were collected with written informed consent. This research was approved by the Institutional Ethical Committee of the Second Affiliated Hospital of Nanchang University.

\section{Construction of TRAF6 plasmid and cell transfection}

The TRAF6 overexpression plasmid TRAF6-pcDNA 3.1 was constructed by inserting a cDNA clone of TRAF6 into the pcDNA3.1 vector. The TRAF6-pcDNA 3.1 plasmid, miR-125b mimic/inhibitor or $\mathrm{NC}$ was directly added to the cells (at 50-60\% confluence) and incubated for $24 \mathrm{~h}$. The cells were transfected with Lipofectamine $^{\circledR} 2000$ (Thermo Fisher Scientific). Finally, the cells were selected with puromycin $(2.5 \mu \mathrm{g} / \mathrm{mL})$. The pcDNA3. 1 vector, miR-125b mimic, miR-125b inhibitor and NC RNA were obtained from GenePharma (Shanghai, China).

\section{Isolation of exosomes}

The exosome pellet was placed on a carbon-coated copper grid, incubated for $5 \mathrm{~min}$ at $37^{\circ} \mathrm{C}$, and then immersed in 2\% phosphotungstic acid solution for $1 \mathrm{~min}$. After washing with PBS, the preparations were captured using a transmission electron microscope (TEM; JEOL, Akishima, Japan) [34]. In addition, the efficiency of exosome isolation was further confirmed by western blotting with three exosome-specific molecular markers: TSG101, CD81 and CD63 (Abcam, Cambridge, UK).

\section{HKCs co-cultured with MSC-Exos}

In brief, $5 \times 10^{4} \mathrm{HKCs}$ were seeded as previously described [35]. In addition, MSCs were first cultured for $48 \mathrm{~h}$. Then, the cell supernatants were collected to isolate exosomes, which were placed in the lower chamber and cocultured with HKCs for $48 \mathrm{~h}$. 


\section{Reverse transcription-quantitative polymerase chain reaction ( $q R T-P C R)$}

Total RNA was extracted from HKCs or MSC-Exos using TRIzol reagent (TaKaRa, Tokyo, Japan). Firststrand cDNA was synthesized by the PrimeScript RT reagent Kit (Takara). RT-qPCR was performed in an ABI7500 system using SYBR Green methods. RT-qPCR was performed in triplicate with the following protocol: $2 \mathrm{~min}$ at $95^{\circ} \mathrm{C}$, followed by 35 cycles $\left(30 \mathrm{~s}\right.$ at $95^{\circ} \mathrm{C}$ and $45 \mathrm{~s}$ at $60^{\circ} \mathrm{C}$ ). The specific primers used were miR-125b F: 5'-GGGGAACATTCAACGCTGT-3', R: 5'-CTCAAC TGGTGTCGTGGAGTC-3'; U6, F: 5'-CTCGCTTCGGC AGCACAT-3', R: 5'-AACGCTTCACGAATTTGCGT-3'; TRAF6 F: 5'-GGGGAACATTCAACGCTGT-3', R: 5'-C TCAACTGGTGTCGTGGAGTC-3'; $\beta$-actin, F: 5'-CAT CATCCCTGCCTCTACTGG-3'， R: 5'-GTGGGTGTCG CTGTTGAAGTC-3'. The $2^{-\Delta \Delta C t}$ method was used to quantify the data.

\section{Dual-luciferase reporter assay}

The 3'-UTR of TRAF6 containing the putative binding sites of miR-125b was synthetized and obtained from Sangon Biotech (Shanghai, China) and then cloned into the pmirGLO Dual-Luciferase Expression Vector (Promega, Madison, WI, USA) to construct the wild-type or mutation-type reporter vector TRAF6 (WT/MT). TRAF6 (WT/MT) was transfected into HKCs together with the control, vector-control (NC) or miR-125b mimic using Lipofectamine 2000 (Thermo Fisher Scientific) according to the manufacturer's instructions. The relative luciferase activity was analysed by the Dual-Glo Luciferase Assay System (Promega).

\section{Western-blot detection}

Total protein was isolated from HKCs by using RIPA buffer. The protein concentration was quantified by a BCA protein kit (Thermo Fisher Scientific). Then, the proteins were separated with SDS-PAGE gel (10\%) and transferred to PVDF membranes. After that, the membranes were incubated with primary antibodies after blocking with $3 \%$ nonfat milk for $1 \mathrm{~h}$. Subsequently, the membranes were incubated with secondary anti-rabbit antibodies $(1: 5,000)$ for $1 \mathrm{~h}$. Finally, the membranes were visualized by an enhanced chemiluminescence (ECL) kit (Thermo Fisher Scientific) and scanned by using an Odyssey Imaging System, and the data were analysed with ImageJ software. Each experiment was repeated three times (Supplementary Fig. 1). The primary antibodies were as follows: anti-TRAF6 (ab33915, 1:2,000), anti-LC3 I (ab192890, 1:2,000), anti-LC3 II (ab63817, 1:1,000), anti-p62 (ab109012, 1:10,000), antiAkt (ab8805, 1:1,000), anti-p-Akt (ab38449, 1:1,000) and anti- $\beta$-actin (ab8227, 1:1,000). $\beta$-actin was used as an internal control. All the antibodies were purchased from Abcam (Cambridge, UK).

\section{Cell apoptosis analysis}

Flow cytometry was conducted using an Annexin VFITC/PI apoptosis detection kit (MA0220, Dalian Meilun Biotech Co., Ltd., Dalian, China). HKCs $\left(2 \times 10^{5}\right.$ cell $/ \mathrm{mL}$ ) were subjected to a 5 -min centrifugation at 500 $g$. The cells were resuspended in $195 \mu \mathrm{L}$ binding buffer, incubated in the dark with $5 \mu \mathrm{L}$ Annexin V-FITC for 10 min and $10 \mu \mathrm{L}$ PI $(20 \mu \mathrm{g} / \mathrm{mL})$ for $5 \mathrm{~min}$ and detected by a flow cytometer.

\section{Statistical analysis}

All the experiments are expressed as the mean \pm standard error (SD). RT-qPCR, flow cytometry and western blotting were repeated in triplicate. One-way analysis of variance (ANOVA) and Tukey's tests were carried out for multiple group comparisons. $P<0.05$ was considered statistically significant. In addition, regression analysis was performed to analyse the correlation.

\section{Results}

\section{MiR-125b was downregulated in DN, while TRAF6 was upregulated}

To investigate the role of miR-125b and TRAF6 in DN, RT-qPCR was performed. As shown in Fig. 1A, the expression of miR-125b was significantly downregulated in DN tissues compared to normal tissues. In contrast, the level of TRAF6 in DN tissues was much higher than that in normal tissues (Fig. 1B). In addition, miR-125b was negatively correlated with TRAF6 (Fig. 1C). Taken together, miR-125b was downregulated in $\mathrm{DN}$, while TRAF6 was upregulated.

\section{Exosomes were successfully isolated from MSCs}

To assess the function of MSC-Exos in DN, MSCExos were isolated. The exosomes were approximately 50-100 nm in diameter (Fig. 2A). In addition, exosomal markers (CD81, CD63 and TSG101) were highly expressed in the exosomes (Fig. 2B). Altogether, exosomes were stably isolated from MSCs and had high purity.

\section{MSC-Exos inhibited HKCs apoptosis by inducing autophagy}

To detect gene expression, qRT-PCR was performed. As demonstrated in Fig. 3A, the expression of miR-125b in HKCs was downregulated by HG, while it was upregulated in MSC-Exos. In addition, the HG-induced decrease in miR-125b expression in HKCs was reversed when the HKCs were cocultured with MSC-Exos (Fig. 
A

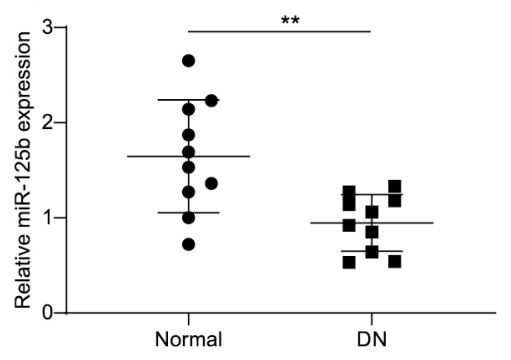

B

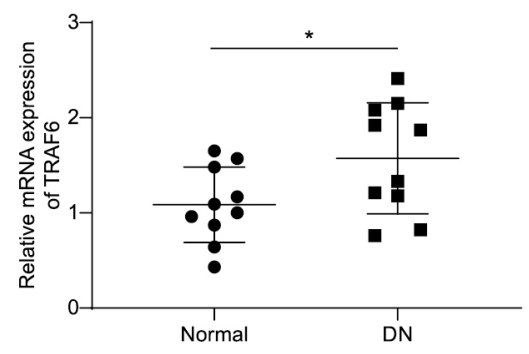

C

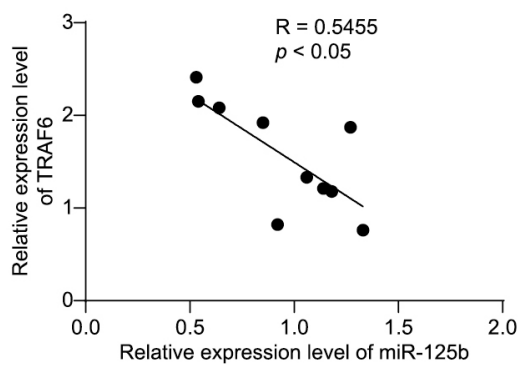

Fig. 1 MiR-125b was downregulated in DN tissues, while TRAF6 was upregulated. (A) The expression of miR-125b in DN tissues or adjacent normal tissues was detected by RT-qPCR. (B) The expression of TRAF6 in DN tissues or adjacent normal tissues was detected by RT-qPCR. (C) Regression analysis was performed to investigate the correlation between miR-125b and TRAF6. $n=$ 15. * $p<0.05, * * p<0.01$.

3A). However, mannitol or LG had a very limited effect on miR-125b expression (Fig. 3A). Moreover, the expression of miR-125b in HKCs was notably upregulated in the presence of MSC-Exos, while this phenomenon was obviously reversed by GW4869 (exosome inhibitor) (Fig. 3B). In addition, HG notably decreased LC3 II/LC3 I expression in HKCs (Fig. 3C). In contrast, the p62 level in HKCs was upregulated by HG (Fig. 3C). However, the effect of $\mathrm{HG}$ on these proteins was partially rescued by MSC-Exos, and the effect of MSC-Exos was also reversed by Baf A1 (Fig. 3C). Furthermore, HG-induced HKCs apoptosis was significantly inhibited when the HKCs were cocultured with MSC-Exos, while LG or mannitol limited cell apoptosis (Fig. 3D). On the other hand, the miR-125b levels in MSC-Exos were greatly reduced when the cells were incubated with the miR-125b inhibitor (Fig. 3E). When HG-treated HKCs were cocultured with MSC-Exos with reduced miR-125b levels, the miR-125b levels in HKCs did not increase (Fig. 3F). Furthermore, inhibiting miR-125b did not change the autophagy and apoptosis of HKCs (Fig. 3G$3 \mathrm{H})$. Taken together, MSC-Exos induced autophagy and inhibited apoptosis in HKCs treated with HG by transferring miR-125b.

\section{MiR-125b directly targeted TRAF6 in HKCs}

To detect the expression of TRAF6 in HKCs, as shown in Fig. 4A and 4B, TRAF6 expression in HGtreated HKCs was dramatically downregulated in the presence of MSC-Exos. These results were partially rescued by miR-125b inhibitors. Additionally, online prediction by TargetScan 7.2 found that the 3'UTR of TRAF6 contained a direct binding site for miR-125b (Fig. 4C). Moreover, decreased luciferase activity was present in HKCs following cotransfection with wtTRAF6 and miR-125b mimic, and the luciferase activity increased after cotransfection with miR-125b inhibitor.
A

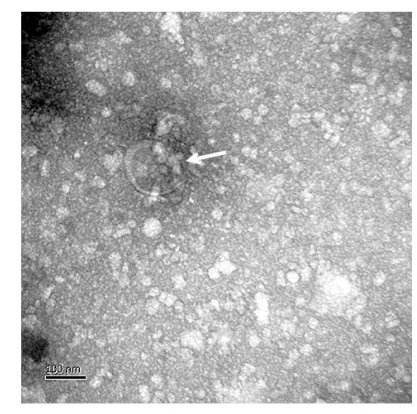

B

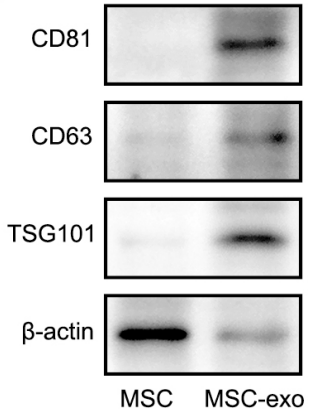

Fig. 2 Exosomes were successfully isolated from MSCs. (A) Exosome pellets were placed on a carbon-coated copper grid, incubated for $5 \mathrm{~min}$ at $37^{\circ} \mathrm{C}$, and then immersed in $2 \%$ phosphotungstic acid solution for $1 \mathrm{~min}$. After washing with PBS, TEM micrographs were captured. (B) The expression of CD81, CD63, TSG101 and $\beta$-actin in MSC-Exos or MSC cell lysates was assessed by western blot. $n=3$. ${ }^{*} p<0.05, * * p<0.01, * * * p<0.001$.

However, when the miR-125b mimic or inhibitor was cotransfected with mut-TRAF6, the luciferase activity in HKCs remained unchanged. (Fig. 4D). In addition, the expression of TRAF6 was increased in HKCs after overexpression of TRAF6 and coculture with MSC-Exos (Fig. 4E and 4F). After overexpressing TRAF6 in HKCs, autophagy in HKCs was significantly decreased (Fig. 4G). In addition, the antiapoptotic effect of MSC-Exos on HG-treated HKCs was partially reversed when the cells were transfected with the TRAF6 overexpression vector (Fig. 4H). In summary, miR-125b from MSCExos reversed the HG-induced autophagy and apoptosis of HKCs by targeting TRAF6.

\section{miR-125b carried by MSC-Exos suppressed the development of DN via the TRAF6/Akt axis}

To explore the mechanism by which MSC-Exos medi- 
A

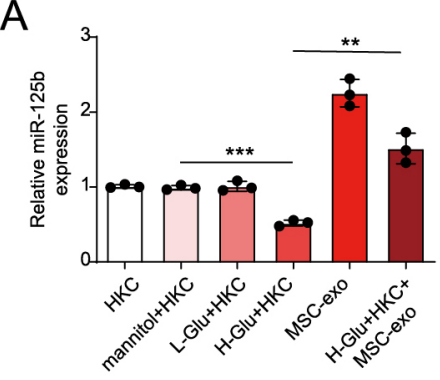

B

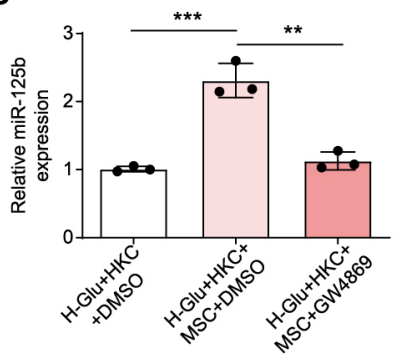

E

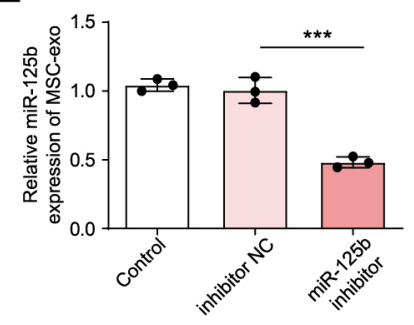

C
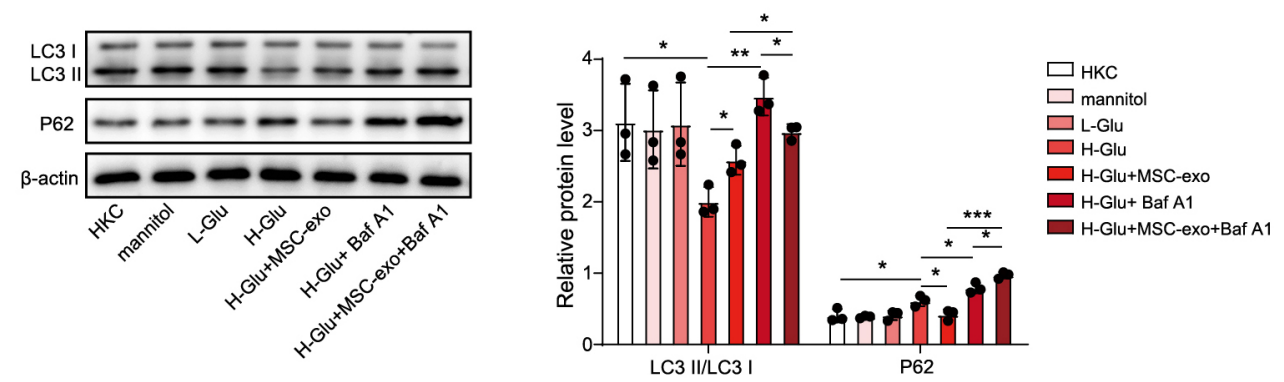

D
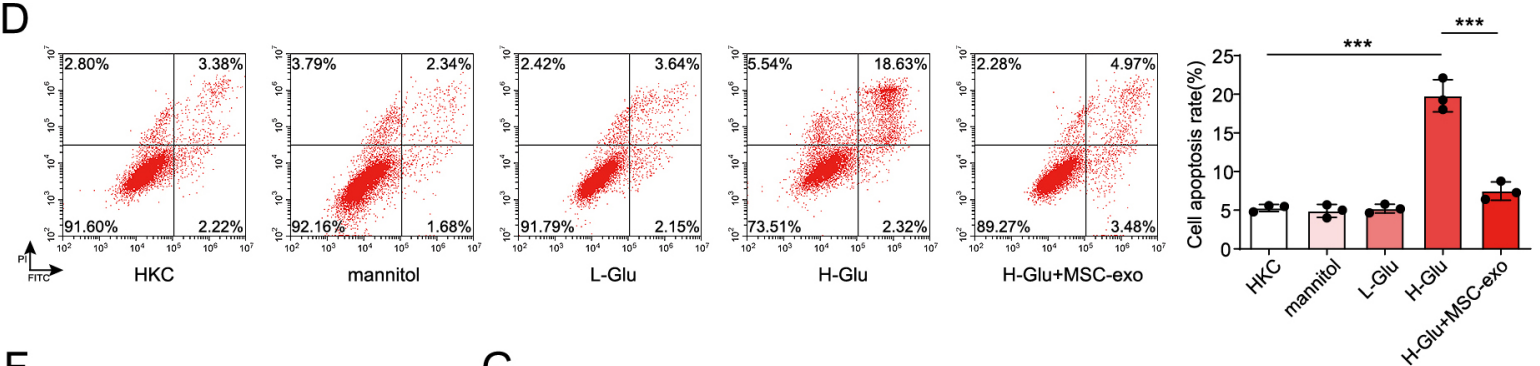

F

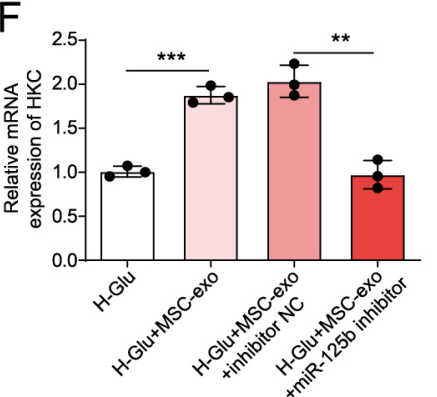

G
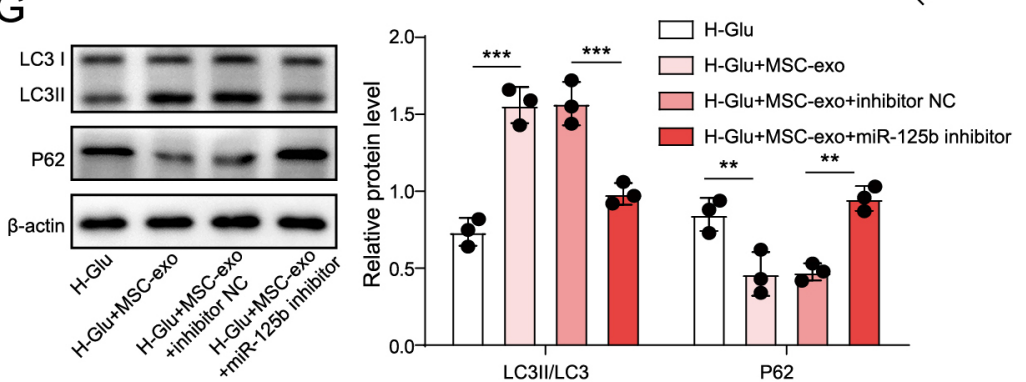

$\mathrm{H}$
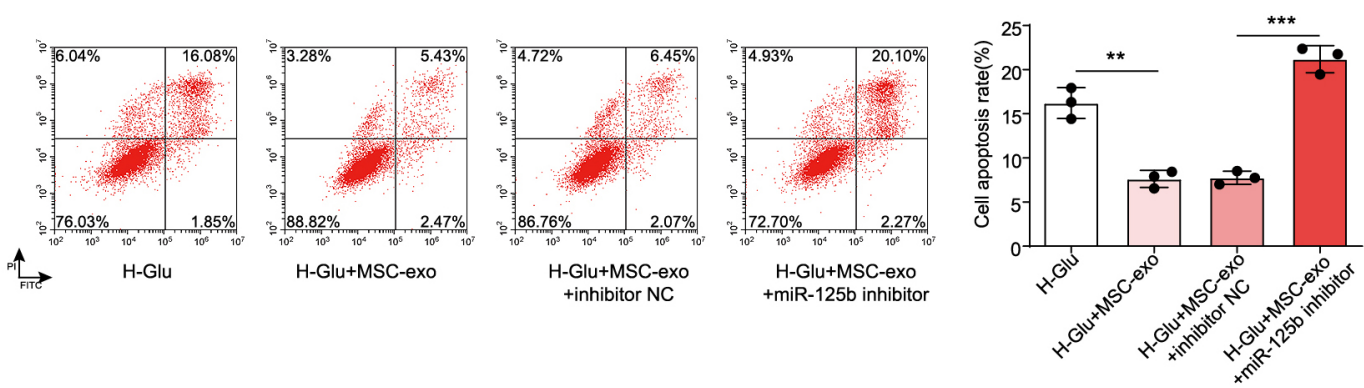

Fig. 3 MSC-Exos inhibited HKCs apoptosis by inducing autophagy. (A) HG-treated HKCs were cocultured with MSC-Exos. MiR-125b expression in HKCs was measured by qRT-PCR. (B) MSCs were treated with GW4869 and cocultured with HG-treated HKCs. Then, the miR-125b levels in the HKCs were detected by qRT-PCR. (C) The LC3 I, LC3 II and p62 levels in HKCs were measured by western blot. The relative expression levels were quantified by normalizing to $\beta$-actin. (D) Flow cytometry was performed to test cell apoptosis. (E) MSCs were transfected with NC or miR-125b inhibitor. Exosomes were derived from MSCs. Then, miR-125b levels in MSC-Exos were analysed by qRT-PCR. (F) MSCs were transfected with the miR-125b inhibitor, and exosomes were derived from the MSCs. Then, HG-induced HKCs were cocultured with MSC-Exos. MiR-125b expression in HKCs was detected by qRT-PCR. (G) LC3 I, LC3 II and p62 expression in HKCs was measured by western blot. The relative levels were quantified by normalization to $\beta$-actin. (H) Cell apoptosis was examined by flow cytometry. $n=3$. ${ }^{*} p<0.05$, ** $p<$ $0.01, * * * p<0.001$. 
A

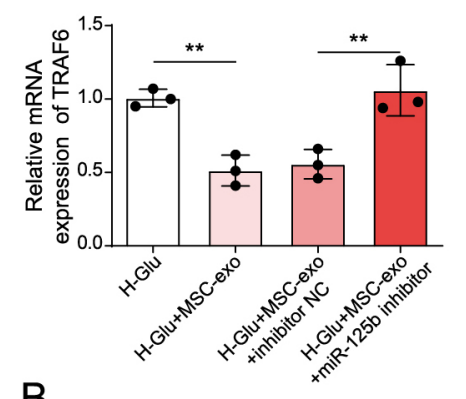

B

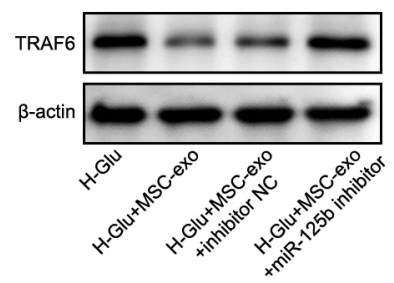

F

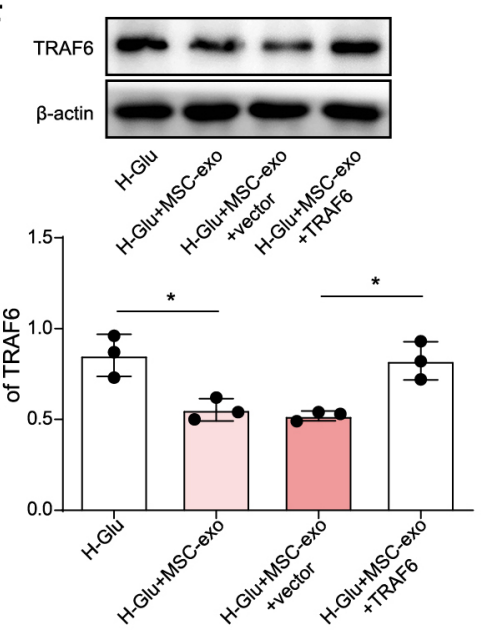

C

TRAF6 5'UTR 3' GUUUCACUAAAUgucacuUgugu hsa-miR-125b-3p 3' GAGGGUUCUCGGACUGAACACU mut TRAF6 5'UTR 3' GUUUCAUAAAUGUCAGAACAGU
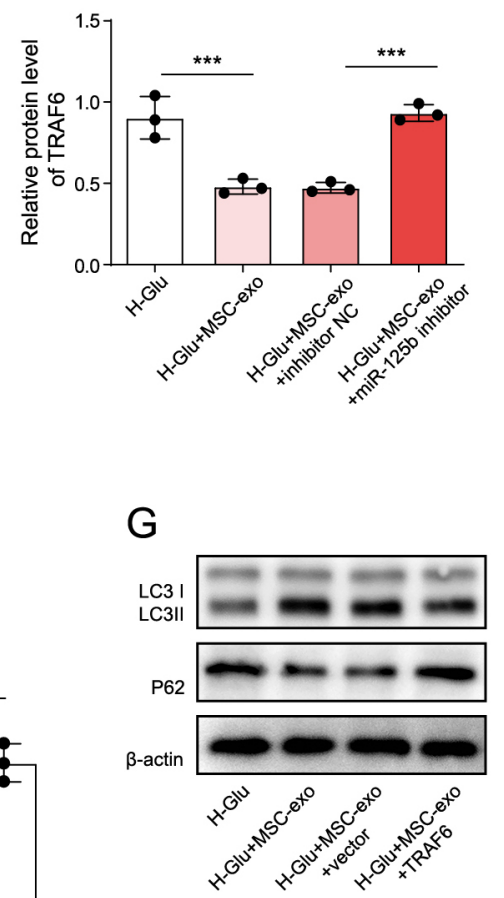

D

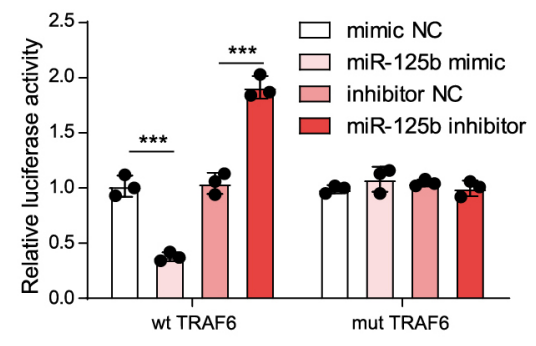

E

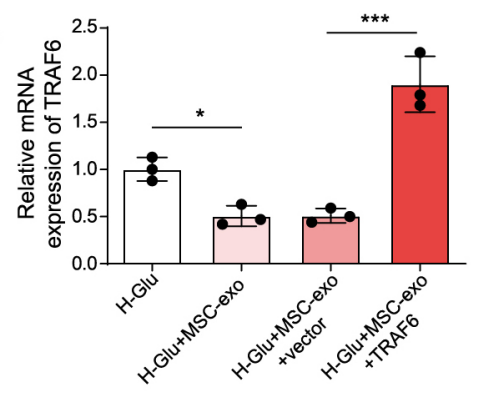

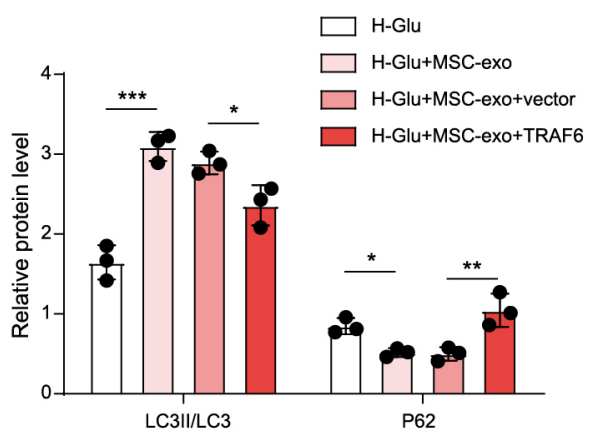

$\mathrm{H}$
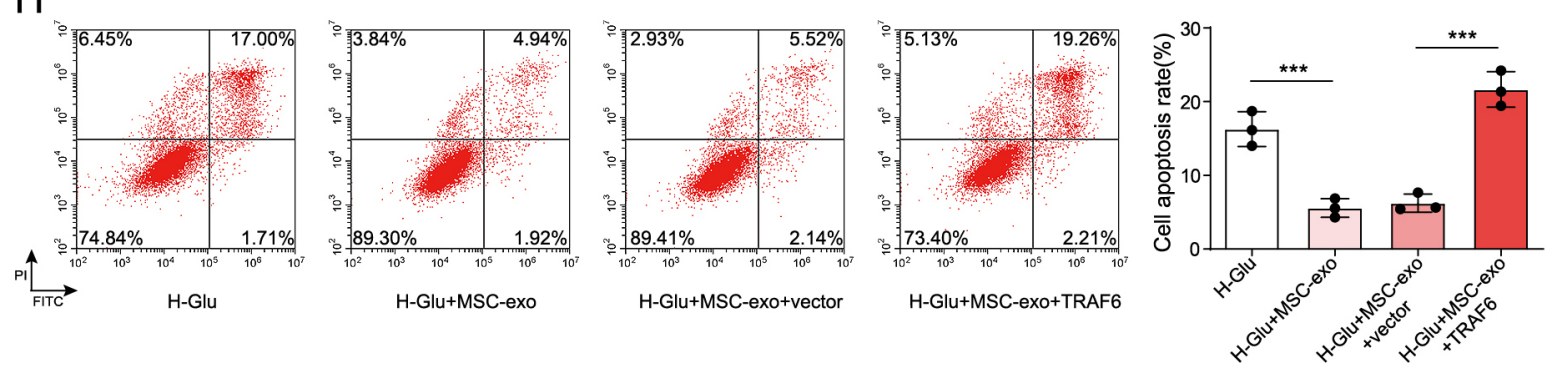

Fig. 4 MiR-125b directly targeted TRAF6 in HKCs. (A) The expression of TRAF6 in HKCs was detected by qRT-PCR. (B) TRAF6 expression in HKCs was measured by western blot. The data were quantified by normalizing to $\beta$-actin. (C) The gene structure of TRAF6 revealed a target site of miR-125b in its 3'UTR. (D) A dual-luciferase reporter assay was used to confirm the correlation between miR-125b and TRAF6. (E) The TRAF6 levels in HKCs were measured by qRT-PCR. (F) TRAF6 expression in HKCs was detected by western blot. The data were quantified by normalizing to $\beta$-actin. (G) LC3 I, LC3 II and p62 expression in HKCs was measured by western blot. The data were quantified by normalization to $\beta$-actin. $(\mathrm{H})$ Cell apoptosis was examined by flow cytometry. $n=3 . * p<0.05, * * p<0.01, * * * p<0.001$.

ated the development of $\mathrm{DN}$, we conducted further investigations. As expected, the levels of phosphorylated Akt in HG-treated HKCs were significantly down- regulated by MSC-Exos, while TRAF6 overexpression reversed the effect of MSC-Exos on the phosphorylation of Akt (Fig. 5A). In HG-induced HKCs cocultured with 
A
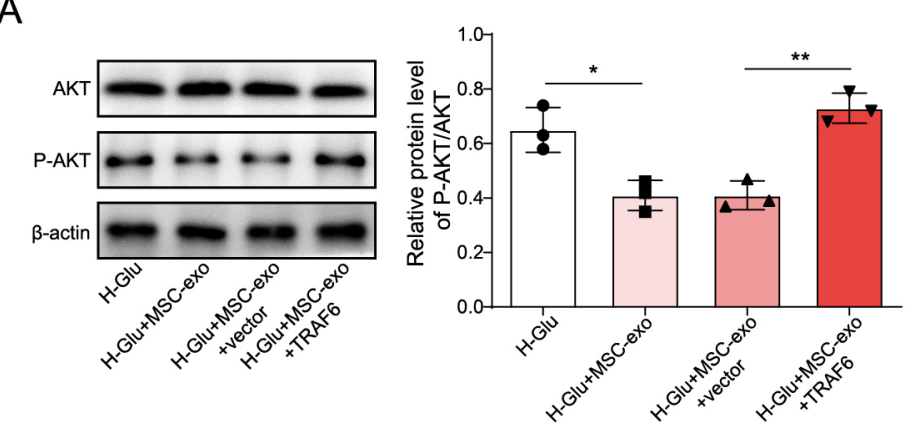

C

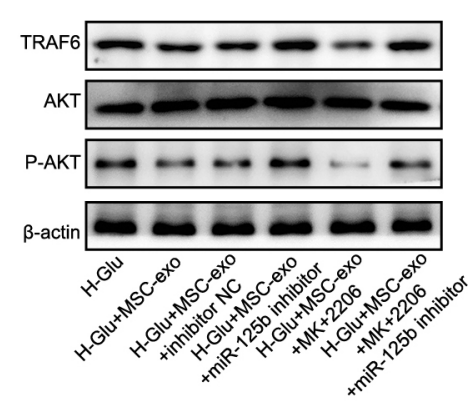

D
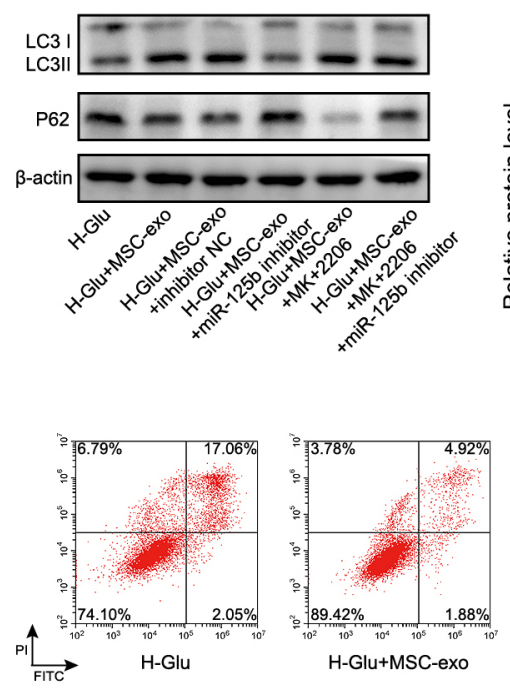
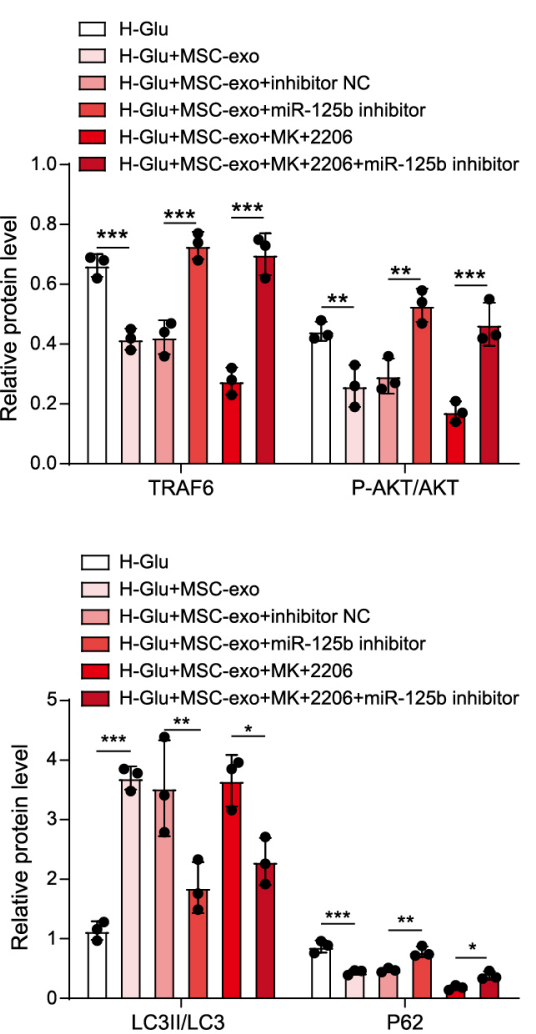
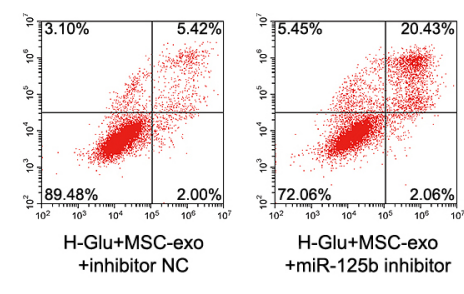

B
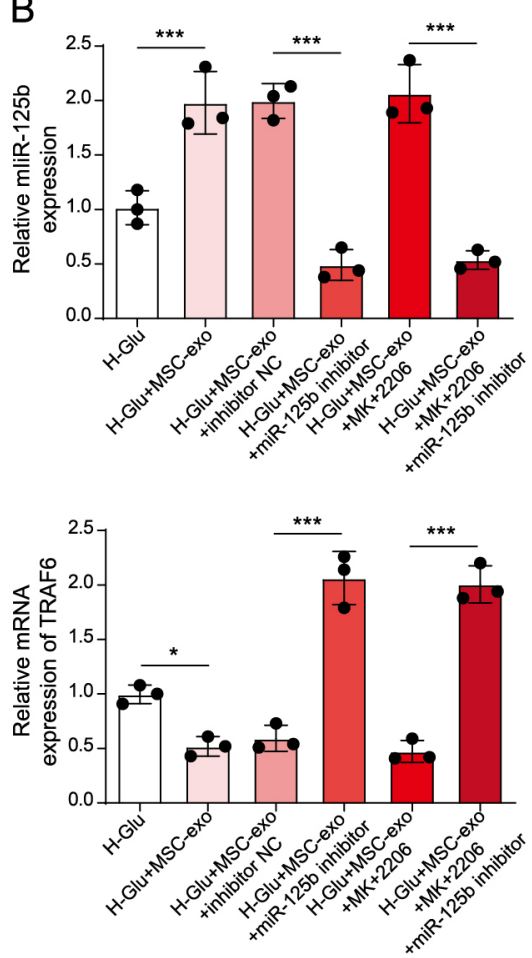

E

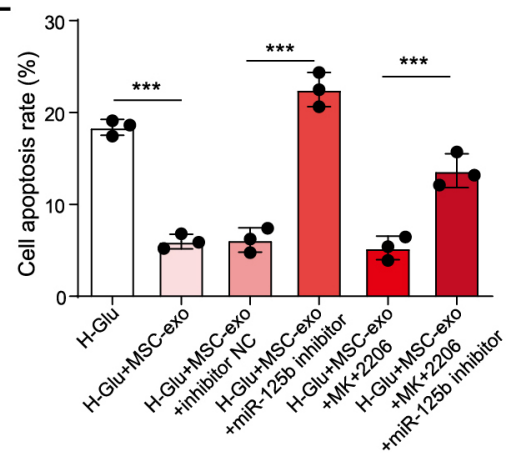

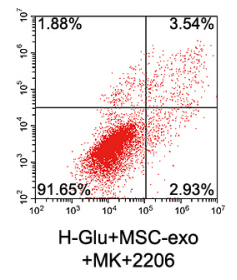

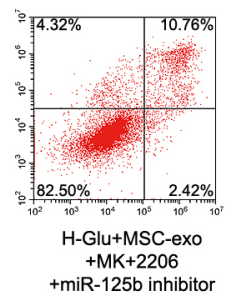

Fig. 5 Exosomes from MSCs expressing miR-125b suppressed the development of DN via regulation of the TRAF6/Akt axis. (A) The Akt and p-Akt levels in HKCs were measured by western blot. (B) The expression of TRAF6 and miR-125b in HKCs was detected by qRT-PCR. (C) Western blotting was used to measure the TRAF6 levels in HKCs, and $\beta$-actin was used for normalization. The expression of Akt and its phosphorylation level were examined by western blotting. (D) The LC3 I, LC3 II and p62 levels in HKCs were assessed by western blot. The data were quantified by normalization to $\beta$-actin. (E) Cell apoptosis was examined by flow cytometry. $n=3 . * p<0.05, * * p<0.01, * * * p<0.001$.

MSC-Exos, the addition of MK-2206 (Akt inhibitor) had a very limited effect on TRAF6 and miR-125b expression (Fig. 5B). However, inhibiting miR-125b significantly increased the phosphorylation of Akt in HKCs treated wtih $\mathrm{HG}$, while this phenomenon was reversed by MK-2206 (Fig. 5C). Additionally, MK-2206 significantly reversed the autophagy inhibitory effect induced by the downregulation of miR-125b (Fig. 5D). In con- 
trast, the downregulation of miR-125b notably induced the apoptosis of HG-induced HKCs cocultured with MSC-Exos, while the effect of miR-125b knockdown on apoptosis was reversed in the presence of MK-2206 (Fig. $5 \mathrm{E})$. In summary, exosomes from MSCs expressing miR-125b suppressed the development of DN via regulation of the TRAF6/Akt axis.

\section{Discussion}

The correlation between HG and miR-125b has been reported. For example, dexmedetomidine could protect neurons from HG-induced injury by inhibiting miR-125b [36]. In addition, knockdown of MALAT1 inhibited the proliferation, migration, tube formation and vascular permeability of hRMECs induced by HG through upregulation of miR-125b [23]. Thus, HG might play a crucial role in the regulation of miR-125b. Thus, the underlying mechanism remains to be further explored.

It has been previously reported that MSC-Exos are involved in multiple diseases [37-39]. In the current research, we found that MSC-Exos inhibited the progression of DN by inducing autophagy. In addition, miR-125b carried by MSC-Exos suppressed the progression of DN via the TRAF6/Akt axis. Our findings increased our understanding of the function of MSCExos in DN, suggesting that MSC-Exos could useful in the study of DN. It has been confirmed that autophagy participates in the progression of $\mathrm{DN}[40,41]$. The induction of autophagy might inhibit the development of DN $[42,43]$. Moreover, exosomes are known to regulate the progression of inflammation induced by DN [16, 44]. Our data were similar to these previous reports. However, the correlation between exosomes and autophagy remains largely unknown. Our research confirmed the relationship between MSC-Exos and autophagy in DN, suggesting that MSC-Exos could be developed as autophagy activators.

The relationship between exosomes and miRNAs was verified. For example, Cai X et al. exosome-transmitted microRNA-133b suppressed bladder cancer cell proliferation by activating dual-specificity protein phosphatase 1 [14]. In addition, exosomal miR-146a derived from MSCs could enhance the sensitivity of ovarian cancer cells to chemotherapy via PI3K/Akt signalling [34]. On the other hand, miRNAs have been confirmed to play key roles in DN $[45,46]$. Moreover, MSC-Exos delivering miR-125b could induce autophagy to attenuate the symptoms of myocardial infarction [24]. Consistently, our study revealed that miR-125b from MSC-Exos could induce autophagy to inhibit HG-induced HKCs apoptosis via Akt signalling, increasing our understanding of the mechanism by which miR-125b mediates the progression of DN.

TRAF6 is known to play key roles in cell growth [47-49]. Furthermore, it has been verified that TRAF6 could induce the progression of DN $[50,51]$. Moreover, it has been previously reported that TRAF6 could modulate Akt signalling in tumours and inflammation $[52,53]$. In addition, inactivation of TRAF6 might lead to cell autophagy [54]. Our results were similar to these previous data. The function of TRAF6 in cell growth may result in a similar phenomenon between these previous studies and our findings. Taken together, our study first suggested that MSC-Exos inhibited the progression of DN via regulation of the TRAF6/Akt axis. On the other hand, it has been revealed that $\mathrm{HG}$ could inactivate the Akt pathway in podocytes [55]. In addition, PC-12 cells treated with $\mathrm{HG}$ exhibited suppressed expression of Akt [56]. Therefore, HG could act as an inhibitor of the Akt pathway, and the potential mechanism by which $\mathrm{HG}$ mediates the Akt pathway needs to be explored in the future.

In summary, MSC-Exos delivering miR-125b inhibited the progression of DN via TRAF6/Akt signalling. Our findings provide a new strategy for the treatment of DN.

\section{Acknowledgements}

We would like to give our sincere gratitude to the reviewers for their constructive comments.

\section{Disclosure Statement}

These authors declared no competing interests in this research.

\section{Declarations}

\section{Funding}

Not Applicable.

\section{Ethical approval}

Not Applicable. This article does not contain any studies with human participants or animals performed by any of the authors.

\section{Consent for publication \\ Not Applicable.}

\section{Availability of data and material}

All data generated or analyzed during this study are included in this article. The datasets used and/or analyzed during the current study are available from the corresponding author on reasonable request. 


\section{Authors' contribution}

Conception and study design: Xia Cai;

Data acquisition: Fang Zou;
Data analysis: Rui Xuan;

Manuscript drafting: Xia Cai;

Manuscript revising: Xiao-Yang Lai
A

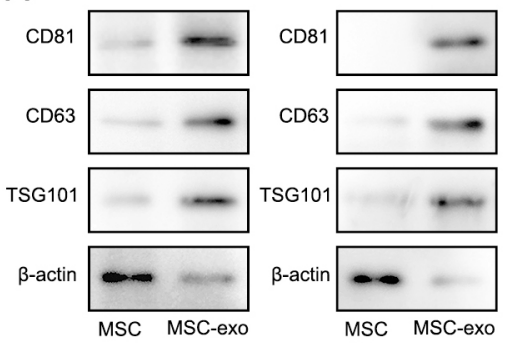

C
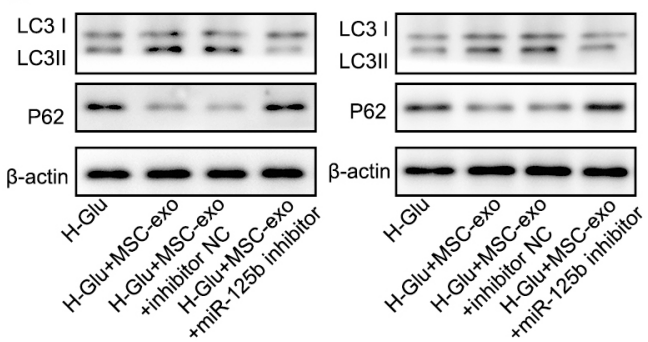

E

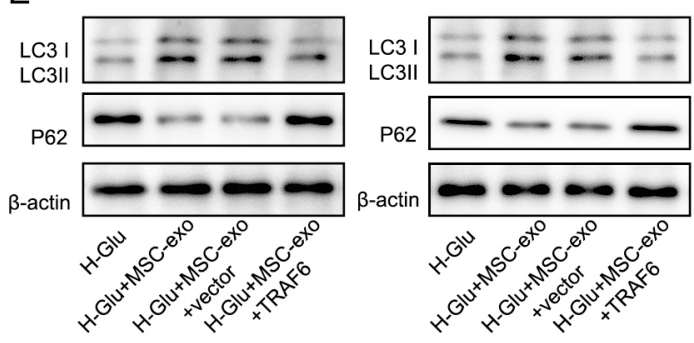

$\mathrm{H}$

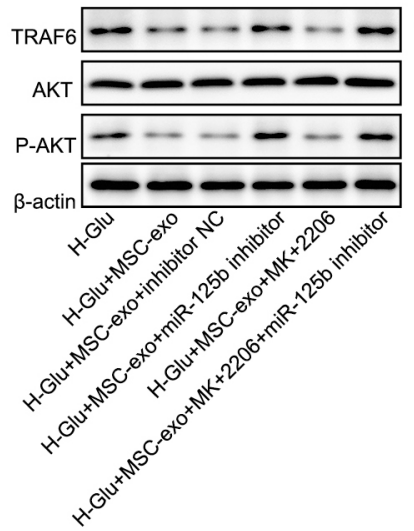

B
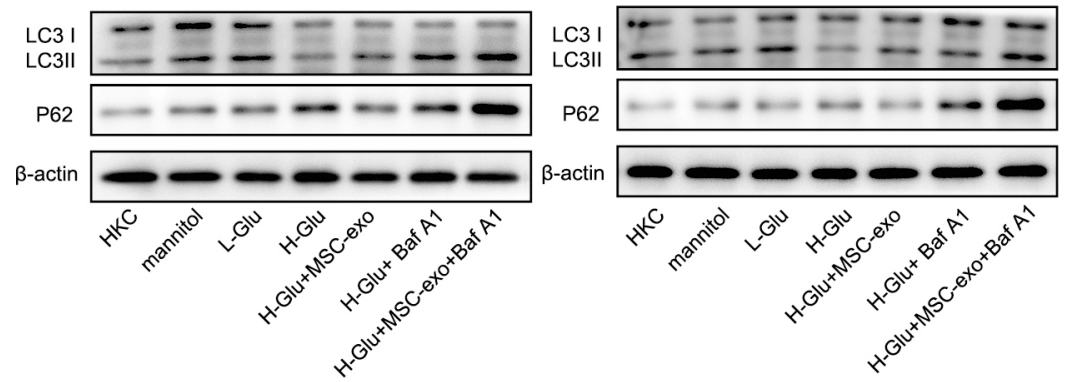

D
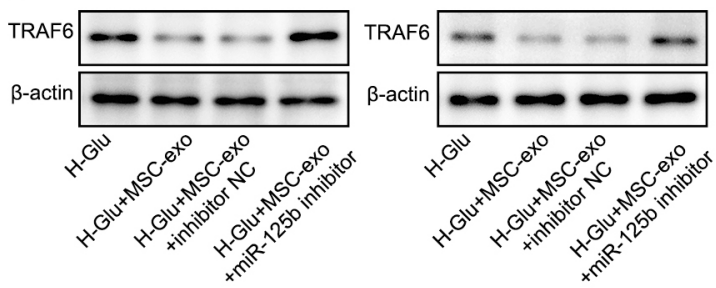

$\mathrm{F}$
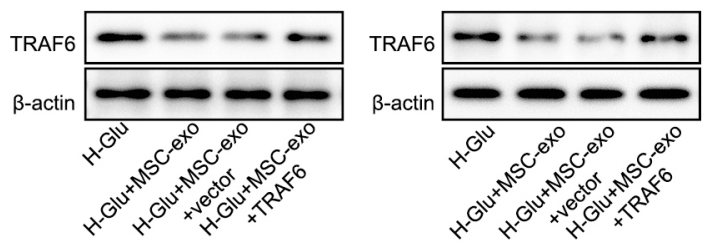

G
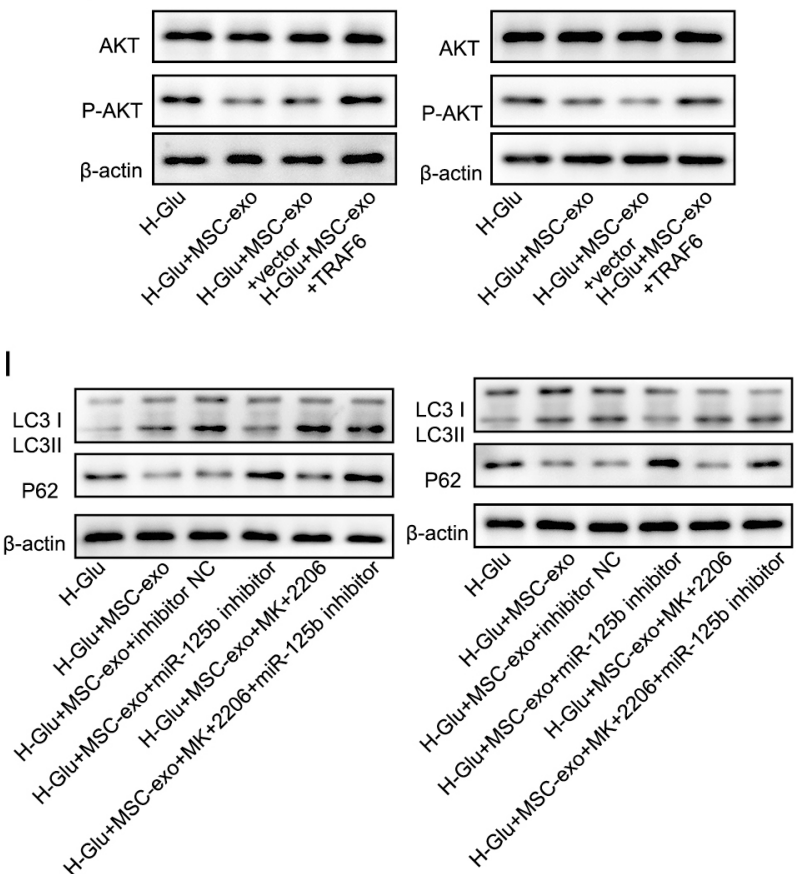

Supplementary Fig. 1 western blot image three times (A) Fig. 2B western blot image. (B) Fig. 3C western blot image. (C) Fig. 3G western blot image. (D) Fig. 4B western blot image. (E) Fig. 4G western blot image. (F) Fig. 4F western blot image. (G) Fig. 5A western blot image. (H) Fig. 5C western blot image. (I) Fig. 5D western blot image. 


\section{References}

1. Tavares G, Venturini G, Padilha K, Zatz R, Pereira AC, et al. (2018) 1,5-anhydroglucitol predicts CKD progression in macroalbuminuric diabetic kidney disease: results from non-targeted metabolomics. Metabolomics 14: 39.

2. Han W, Ma Q, Liu Y, Wu W, Tu Y, et al. (2019) Huangkui capsule alleviates renal tubular epithelialmesenchymal transition in diabetic nephropathy via inhibiting NLRP3 inflammasome activation and TLR4/NFkappaB signaling. Phytomedicine 57: 203-214.

3. Kamiya A, Hara T, Tsuda M, Tsuru E, Kuroda Y, et al. (2019) 5-aminolevulinic acid with ferrous iron improves early renal damage and hepatic steatosis in high fat dietinduced obese mice. J Clin Biochem Nutr 64: 59-65.

4. Xue H, Li P, Luo Y, Wu C, Liu Y, et al. (2019) Salidroside stimulates the Sirt1/PGC-1alpha axis and ameliorates diabetic nephropathy in mice. Phytomedicine 54: 240 247.

5. Qu G, Zhao X, Jiang G (2018) Comment on "In Vivo Neuroimaging of Exosomes Using Gold Nanoparticles". ACS Nano 12: 11717-11718.

6. Santulli G (2018) Exosomal microRNA: the revolutionary endogenous Innerspace nanotechnology. Sci Transl Med 10: eaav9141.

7. Fathollahi A, Hashemi SM, Haji Molla Hoseini M, Yeganeh F (2019) In vitro analysis of immunomodulatory effects of mesenchymal stem cell- and tumor cell -derived exosomes on recall antigen-specific responses. Int Immunopharmacol 67: 302-310.

8. Perets N, Hertz S, London M, Offen D (2018) Intranasal administration of exosomes derived from mesenchymal stem cells ameliorates autistic-like behaviors of BTBR mice. Mol Autism 9: 57.

9. Kim S, Lee SK, Kim H, Kim TM (2018) Exosomes secreted from induced pluripotent stem cell-derived mesenchymal stem cells accelerate skin cell proliferation. Int $J$ Mol Sci 19: 3119.

10. Wang D, Gao B, Yue J, Liu F, Liu Y, et al. (2019) Exosomes from mesenchymal stem cells expressing miR-125b inhibit neointimal hyperplasia via myosin IE. $J$ Cell Mol Med 23: 1528-1540.

11. Zhao J, Li X, Hu J, Chen F, Qiao S, et al. (2019) Mesenchymal stromal cell-derived exosomes attenuate myocardial ischaemia-reperfusion injury through miR-182regulated macrophage polarization. Cardiovasc Res 115: 1205-1216.

12. Nozohouri S, Vaidya B, Abbruscato TJ (2020) Exosomes in ischemic stroke. Curr Pharm Des 26: 5533-5545.

13. Resaz R, Cangelosi D, Morini M, Segalerba D, Mastracci $\mathrm{L}$, et al. (2020) Circulating exosomal microRNA as potential biomarkers of hepatic injury and inflammation inGlycogen storage disease type 1a. Dis Model Mech 13: dmm043364.

14. Cai X, Qu L, Yang J, Xu J, Sun L, et al. (2020) Exosometransmitted microRNA-133b inhibited bladder cancer proliferation by upregulating dual-specificity protein phosphatase 1. Cancer Med 9: 6009-6019.

15. Lv J, Zhao HP, Dai K, Cheng Y, Zhang J, et al. (2020) Circulating exosomal miRNAs as potential biomarkers for Barrett's esophagus and esophageal adenocarcinoma. World J Gastroenterol 26: 2889-2901.

16. Zhu M, Sun X, Qi X, Xia L, Wu Y (2020) Exosomes from high glucose-treated macrophages activate macrophages andinduce inflammatory responses via NF-kappaB signaling pathway in vitro and in vivo. Int Immunopharmacol 84: 106551.

17. Kim H, Bae YU, Jeon JS, Noh H, Park HK, et al. (2019) The circulating exosomal microRNAs related to albuminuria in patients with diabetic nephropathy. $J$ Transl Med 17: 236.

18. Zang J, Maxwell AP, Simpson DA, McKay GJ (2019) Differential expression of urinary exosomal microRNAs miR-21-5p and miR-30b-5p in individuals with diabetic kidney disease. Sci Rep 9: 10900.

19. Wang B, Yao K, Huuskes BM, Shen HH, Zhuang J, et al. (2016) Mesenchymal stem cells deliver exogenous microRNA-let7c via exosomes to attenuate renal fibrosis. Mol Ther 24: 1290-1301.

20. Lee SE, Jang JE, Kim HS, Jung MK, Ko MS, et al. (2019) Mesenchymal stem cells prevent the progression of diabetic nephropathy by improving mitochondrial function in tubular epithelial cells. Exp Mol Med 51: 77.

21. Xu J, Xiang P, Liu L, Sun J, Ye S (2020) Metformin inhibits extracellular matrix accumulation, inflammation and proliferation of mesangial cells in diabetic nephropathy by regulating H19/miR-143-3p/TGF-beta1 axis. $J$ Pharm Pharmacol 72: 1101-1109.

22. Wang F, Gao X, Zhang R, Zhao P, Sun Y, et al. (2019) LncRNA TUG1 ameliorates diabetic nephropathy by inhibiting miR-21 to promote TIMP3-expression. Int $J$ Clin Exp Pathol 12: 717-729.

23. Liu P, Jia SB, Shi JM, Li WJ, Tang LS, et al. (2019) LncRNA-MALAT1 promotes neovascularization in diabetic retinopathy through regulating $\mathrm{miR}-125 \mathrm{~b} / \mathrm{VE}-$ cadherin axis. Biosci Rep 39: BSR20181469.

24. Zhu LP, Tian T, Wang JY, He JN, Chen T, et al. (2018) Hypoxia-elicited mesenchymal stem cell-derived exosomes facilitates cardiac repair through miR-125bmediated prevention of cell death in myocardial infarction. Theranostics 8: 6163-6177.

25. Liu M, Yan M, Lv H, Wang B, Lv X, et al. (2020) Macrophage K63-linked ubiquitination of YAP promotes its nuclear localization and exacerbates atherosclerosis. Cell Rep 32: 107990.

26. Zhou B, Li Q, Wang J, Chen P, Jiang S (2019) Ellagic acid attenuates streptozocin induced diabetic nephropathy via the regulation of oxidative stress and inflammatory signaling. Food Chem Toxicol 123: 16-27.

27. Guo C, Zhang L, Nie L, Zhang N, Xiao D, et al. (2016) 
Association of polymorphisms in the MyD88, IRAK4 and TRAF6 genes and susceptibility to type 2 diabetes mellitus and diabetic nephropathy in a southern Han Chinese population. Mol Cell Endocrinol 429: 114-119.

28. Yang F, Qu Q, Zhao C, Liu X, Yang P, et al. (2020) Paecilomyces cicadae-fermented Radix astragali activates podocyte autophagy by attenuating PI3K/AKT/mTOR pathways to protect against diabetic nephropathy in mice. Biomed Pharmacother 129: 110479.

29. Xu J, Deng Y, Wang Y, Sun X, Chen S, et al. (2020) SPAG5-AS1 inhibited autophagy and aggravated apoptosis of podocytes via SPAG5/AKT/mTOR pathway. Cell Prolif 53: e12738.

30. Stephens E, Roy M, Bisson M, Nguyen HD, Scott MS, et al. (2020) Osteoclast signaling-targeting miR-146a-3p and miR-155-5p are downregulated in Paget's disease of bone. Biochim Biophys Acta Mol Basis Dis 1866: 165852.

31. Shao J, Ding Z, Peng J, Zhou R, Li L, et al. (2020) MiR-146a-5p promotes IL-1beta-induced chondrocyte apoptosis through the TRAF6-mediated NF-kB pathway. Inflamm Res 69: 619-630.

32. Shi YF, Yu DJ, Jiang CY, Wang XJ, Zhu YP, et al. (2018) TRAF6 regulates proliferation of stromal cells in the transition and peripheral zones of benign prostatic hyperplasia via Akt/mTOR signaling. Prostate 78: 193-201.

33. Gurung S, Ulrich D, Sturm M, Rosamilia A, Werkmeister JA, et al. (2020) Comparing the effect of TGF-beta receptor inhibition on human perivascular mesenchymal stromal cells derived from endometrium, bone marrow and adipose tissues. J Pers Med 10: 261.

34. Qiu L, Wang J, Chen M, Chen F, Tu W (2020) Exosomal microRNA146a derived from mesenchymal stem cells increases the sensitivity of ovarian cancer cells to docetaxel and taxane via a LAMC2mediated PI3K/Akt axis. Int J Mol Med 46: 609-620.

35. Zhang W, Xing L, Xu L, Jin X, Du Y, et al. (2019) Nudel involvement in the high-glucose-induced epithelialmesenchymal transition of tubular epithelial cells. $\mathrm{Am} \mathrm{J}$ Physiol Renal Physiol 316: F186-F194.

36. Hou X, Xu F, Zhang C, Shuai J, Huang Z, et al. (2020) Dexmedetomidine exerts neuroprotective effects during high glucose-induced neural injury by inhibiting miR125b. Biosci Rep 40: BSR20200394.

37. Lee S, Kim S, Chung H, Moon JH, Kang SJ, et al. (2020) Mesenchymal stem cell-derived exosomes suppress proliferation of $\mathrm{T}$ cells by inducing cell cycle arrest through p27kip1/Cdk2 signaling. Immunol Lett 225: 16-22.

38. Liu Z, Wu C, Zou X, Shen W, Yang J, et al. (2020) Exosomes derived from mesenchymal stem cells inhibit neointimal hyperplasia by activating the Erk1/2 signalling pathway in rats. Stem Cell Res Ther 11: 220.

39. Zhang S, Liu X, Ge LL, Li K, Sun Y, et al. (2020) Mesenchymal stromal cell-derived exosomes improve pulmonary hypertension through inhibition of pulmonary vascular remodeling. Respir Res 21: 71.

40. Wen D, Tan RZ, Zhao CY, Li JC, Zhong X, et al. (2020) Astragalus mongholicus Bunge and Panax notoginseng
(Burkill) F.H. Chen formula for renal injury in diabetic nephropathy-in vivo and in vitro evidence for autophagy regulation. Front Pharmacol 11: 732.

41. Jiang XS, Xiang XY, Chen XM, He JL, Liu T, et al. (2020) Inhibition of soluble epoxide hydrolase attenuates renal tubular mitochondrial dysfunction and ER stress by restoring autophagic flux in diabetic nephropathy. Cell Death Dis 11: 385.

42. Zheng XP, Nie Q, Feng J, Fan XY, Jin YL, et al. (2020) Kidney-targeted baicalin-lysozyme conjugate ameliorates renal fibrosis in rats with diabetic nephropathy induced by streptozotocin. BMC Nephrol 21: 174.

43. Khokhar M, Roy D, Modi A, Agarwal R, Yadav D, et al. (2020) Perspectives on the role of PTEN in diabetic nephropathy: an update. Crit Rev Clin Lab Sci 57: 470483.

44. Li H, Rong P, Ma X, Nie W, Chen Y, et al. (2020) Mouse umbilical cord mesenchymal stem cell paracrine alleviates renal fibrosis in diabetic nephropathy by reducing myofibroblast transdifferentiation and cell proliferation and upregulating MMPs in mesangial cells. $J$ Diabetes Res 2020: 3847171.

45. Sun J, Wang J, Lu W, Xie L, Lv J, et al. (2020) MiR-325-3p inhibits renal inflammation and fibrosis by targeting CCL19 in diabetic nephropathy. Clin Exp Pharmacol Physiol 47: 1850-1860.

46. Duan Y, Luo Q, Wang Y, Ma Y, Chen F, et al. (2020) Adipose mesenchymal stem cell-derived extracellular vesicles containing microRNA-26a-5p target TLR4 and protect against diabetic nephropathy. J Biol Chem 295: 12868-12884.

47. Kong R, Gao J, Ji L, Peng Y, Zhang J, et al. (2020) Iguratimod ameliorates rheumatoid arthritis progression through regulating miR-146a mediated IRAK1 expression and TRAF6/JNK1 pathway: an in vivo and in vitro study. Clin Exp Rheumatol Online ahead of print.

48. Ji M, Mei X, Jing X, Xu X, Chen X, et al. (2020) The cooperative complex of Argonaute-2 and microRNA-146a regulates hepatitis $\mathrm{B}$ virus replication through flap endonuclease 1. Life Sci 257: 118089.

49. Tanner EA, Gary MA, Davis AA, Michalik S, McFarlin BK (2020) Alterations in systemic inflammatory response following a half-marathon race with a combined curcumin and pomegranate supplement: a feasibility study. J Diet Suppl Online ahead of print 1-17.

50. Feng Y, Ge Y, Wu M, Xie Y, Wang M, et al. (2020) Long noncoding RNAs regulate inflammation in diabetic peripheral neuropathy by acting as ceRNAs targeting miR-146a-5p. Diabetes Metab Syndr Obes 13: 413-422.

51. Tian S, Wang M, Liu C, Zhao H, Zhao B (2019) Mulberry leaf reduces inflammation and insulin resistance in type 2 diabetic mice by TLRs and insulin signalling pathway. BMC Complement Altern Med 19: 326.

52. Chen H, Fang C, Zhi X, Song S, Gu Y, et al. (2020) Neobavaisoflavone inhibits osteoclastogenesis through blocking RANKL signalling-mediated TRAF6 and c-Src recruitment and NF-kappaB, MAPK and Akt pathways. $J$ 
Cell Mol Med 24: 9067-9084.

53. Hou H, Yang W, Bao S, Cao Y (2020) Epigallocatechin gallate suppresses inflammatory responses by inhibiting toll-like receptor 4 signaling and alleviates insulin resistance in the livers of high-fat-diet rats. J Oleo Sci 69: 479486.

54. Kim MJ, Min Y, Im JS, Son J, Lee JS, et al. (2020) p62 is Negatively implicated in the TRAF6-BECN1 signaling axis for autophagy activation and cancer progression by toll-like receptor 4 (TLR4). Cells 9: 1142.
55. Yan H, Xu F, Xu J, Song MA, Wang K, et al. (2020) Activation of Akt-dependent Nrf2/ARE pathway by restoration of Brg-1 remits high glucose-induced oxidative stress and ECM accumulation in podocytes. J Biochem Mol Toxicol Online ahead of print e22672.

56. Hossain KFB, Hosokawa T, Saito T, Kurasaki M (2020) Amelioration of butylated hydroxytoluene against inorganic mercury induced cytotoxicity and mitochondrial apoptosis in PC12 cells via antioxidant effects. Food Chem Toxicol 146: 111819. 